Revue internationale P.M.E.

Économie et gestion de la petite et moyenne entreprise

\title{
Le fardeau financier de l'informatisation dans la petite entreprise
}

\section{Elisabeth Lefebvre et Louis-A. Lefebvre}

Volume 1, numéro 3-4, 1988

URI : https://id.erudit.org/iderudit/1007888ar

DOI : https://doi.org/10.7202/1007888ar

Aller au sommaire du numéro

Éditeur(s)

Presses de l’Université du Québec

ISSN

0776-5436 (imprimé)

1918-9699 (numérique)

Découvrir la revue

Citer cette note

Lefebvre, E. \& Lefebvre, L.-A. (1988). Le fardeau financier de l'informatisation dans la petite entreprise. Revue internationale P.M.E., 1(3-4), 327-334.

https://doi.org/10.7202/1007888ar d'utilisation que vous pouvez consulter en ligne. 


\title{
Le fardeau financier de l'informatisation dans la petite entreprise ${ }^{*}$
}

\author{
Elisabeth LEFEBVRE** \\ Louis-A. LEFEBVRE** \\ Université du Québec à Montréal
}

\section{Introduction}

L'informatisation des activités de gestion d'une petite entreprise est un choix dont les retombées financières ne peuvent être ignorées. En effet, certains auteurs, tel Delone (1988), ont indiqué que les investissements requis étaient généralement importants compte tenu des contraintes financières auxquelles sont sujettes les petites entreprises. Parmi les obstacles majeursàl'adoption des nouvelles technologies par les petites entreprises, on retient entre autres le coût d'acquisition des équipements (CEC, 1987; OTA, 1985) et le risque financier associé à ces acquisitions (Julien et al., 1988). Mais qu'en est-il réellement du coût relatif de l'informatisation dans la petite entreprise ? Existe-t-il un seuil financier au-delà duquel les entreprises acceptent l'informatisation ? Le chiffre d'affaires serait-il associé à la décision d'informatisation? Le poids financier de l'informatisation est-il plus lourd pour les petites entreprises que pour celles de plus grande taille ? Pour répondre à ces questions, nous nous proposons dans cette note de présenter les résultats d'une recherche conduite auprès de 1708 entreprises québécoises.

- Cette recherche a été rendue possible grâce à des subventions obtenues des organismes suivants: Le Groupe DMR Inc. et ATT Canada. Les auteurs voudraient remercier Manchi Luc et Anny Filiatrault pour leur assistance de recherche.

** Les auteurs sont respectivement membre et directeur du Groupe de recherche en informatisation des entreprises à l'UQAM. Ils enseignent en système d'information. Ils ont publié plusieurs travaux sur le sujet.

(Adresse: Département des sciences administratives, Université du Québec à Montréal, C.P. 8888, Succursale A, Montréal, Québec, H3C 3P8). Manuscrit reçu en décembre 1988.) 


\section{L'enquête}

Une enquête fut menée, par mode de questionnaire d'administration directe, auprès des dirigeants des entreprises membres de l'une des associations suivantes : la Chambre de Commerce du Québec, la Chambre de Commerce de Montréal, le Centre des dirigeants d'entreprises et le Regroupement québécois des entreprises. Au total, 1304 dirigeants de petites entreprises et 404 de moyennes et grandes ont choisi de participer à l'enquête. Aux fins de cette recherche, la petite entreprise est définie comme celle ayant 100 employés ou moins. Cette définition correspond à celle retenue par divers organismes (MIC, 1987; CEC, 1986; OTA, 1985). La répartition des répondants est présentée au tableau 1.

Tab.1. Répartition des entreprises selon la taille (1)

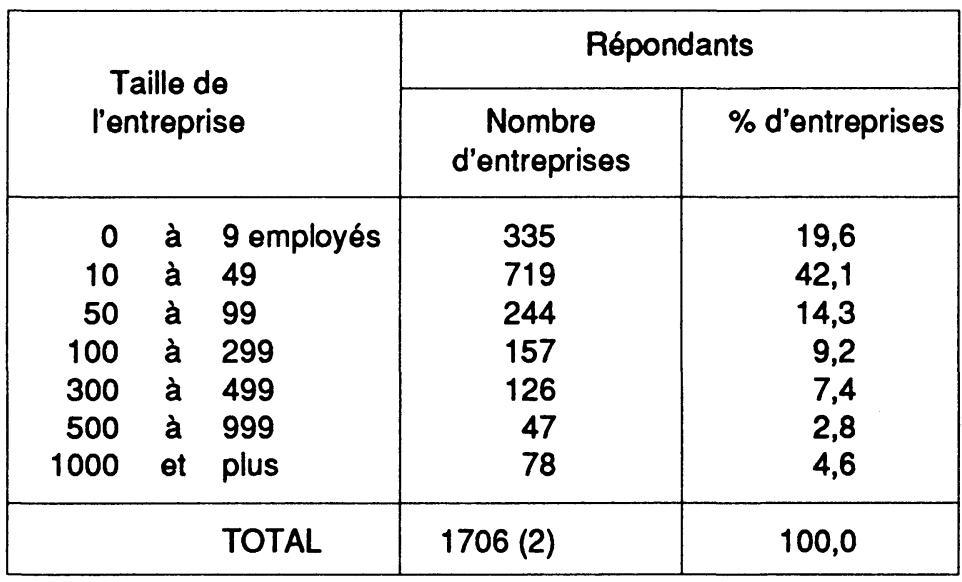

(1) Nous retenons les définitions suivantes: petite entreprise : moins de 100 employés moyenne entreprise : entre 100 et 500 employés grande entreprise : plus de 500 employés

(2) Parmi les 1708 entreprises, 2 entreprises n'ont pu être identifiées à une taille.

\section{Questions de recherche}

Il est généralement reconnu que la taille de l'entreprise est associée à l'adoption des nouvelles technologies (Lefebvre et al., 1986). Or, malgré que l'accessibilité de l'informatique a été grandement accrue dans les petites entreprises suite à l'avènement de la micro informatique, les dépenses afférentes restent cependant importantes pour la majorité d'entre elles (Martin \& McClure, 1983). A 
ce titre, le chiffre d'affaires en tant qu'indicateur de la capacité financière d'une entreprise devrait constituer un facteur important lors de la décision d'informatisation.

Lescoûts de l'informatisation dans une entreprise comprennenten particulier les coûts d'acquisition de l'équipement (quincaillerie et logiciels) et les frais annuels d'exploitation. Les premiers sont proportionnellement plus importants pour la petite entreprise, puisque certains frais fixes associés à l'acquisition d'une configuration informatique doivent être encourus indépendamment de la taille de l'entreprise. Les frais d'exploitation dans les petites entreprises, quant à eux, seraient rarement formalisés ou même existants en raison de caractéristiques propres aux petites entreprises. D'une part, les pratiques de gestion dans ces entreprises étant caractérisées par des préoccupations de court terme (Welsh et White, 1981; DeLone, 1981; Raymond, 1985), certaines dépenses d'exploitation telles l'entretien et la sécurité seraient considérées comme secondaires. D'autre part, le manque chronique de ressources internes à l'entreprise (DeLone, 1988; Lees, 1987) ne permettrait pas de créer et de maintenir une fonction informatique responsable de l'exploitation du système. Nombre d'entre elles doivent donc s'en remettre aux services de fournisseurs de technologie ou encore de consultants externes pour assurer les besoins de formation et d'entretien (Meredith, 1987). Or, le coût de ces services est généralement trop élevé pour que les petites entreprises puissent s'en prévaloir (DeLone, 1988). Enfin, lors de l'introduction de l'informatique dans l'entreprise, l'emphase est mise essentiellement surl'acquisition d'équipements plutôt que sur d'autres frais inhérents tels les coûts de formation (Lees, 1987, Griffith et Dorsman, 1986). Par contre, dans la situation où ces frais annuels d'exploitation seraient existants, nous postulons que tout comme les frais d'acquisition, ces frais annuels seraient proportionnellement plus élevés dans la petite entreprise. Dans cette perspective, il est proposé de vérifier les propositions suivantes portant sur le fardeau financier de l'informatisation dans la petite entreprise :

P1 Le chiffre d'affaires est un facteur significativement associé à la décision d'informatisation d'une petite entreprise et ce, indépendamment de la taille de l'entreprise.

P2 La valeur actuelle relative des équipements informatiques est significativement plus élevée dans les petites entreprises que dans les moyennes et grandes.

P3 La valeur relative de l'investissement prévu en informatisation est significativement plusélevée dans les petites entreprises que dans les moyennes et grandes.

P4 Le budget relatif annuel accordé à la formation du personnel, à l'entretien et à la sécurité informatique est significativement plus élevé dans les petites entreprises que dans les moyennes et grandes. 


\section{Résultats}

Un premier profil des entreprises informatisées, prévoyant s'informatiser et non informatisées est proposé au tableau 2. Les résultats démontrent clairement que la taille est un facteur associé à l'informatisation d'une entreprise. Nous remarquons d'abord que les entreprises de plus de 100 employés ont largement adopté les technologies informatiques. La situation est tout autre en ce qui concerne les petites entreprises puisque pour bon nombre d'entre elles, notamment les plus petites, l'adoption est moins répandue.

Si la taille est un facteur important, qu'en est-il du chiffre d'affaires au sein d'une même taille d'entreprises ? L'analyse des résultats nous permet de constater des différences significatives dans les chiffres d'affaires selon que les entreprises sont informatisées, prévoient l'être, ou ne le sont pas. Ainsi, pour les entreprises de 50 à 99 employés, le chiffre d'affaires médian est de 7,5 millions de dollars pour celles qui sont informatisées, alors qu'il est de 6 millions pour celles qui prévoient s'informatiser, et de seulement 3 millions pour celles qui ne le sont pas (tableau 3).

Tab. 2. Répartition des entreprises selon leur état d'informatisation

\begin{tabular}{|c|c|c|c|c|c|c|c|c|c|c|}
\hline \multirow{3}{*}{\multicolumn{3}{|c|}{$\begin{array}{c}\text { Taille de } \\
\text { l'entreprise }\end{array}$}} & \multicolumn{8}{|c|}{ Entreprises } \\
\hline & & & \multicolumn{2}{|c|}{$\begin{array}{l}\text { Ne prévoyant pas } \\
\text { s'informatiser }\end{array}$} & \multicolumn{2}{|c|}{$\begin{array}{l}\text { Prévoyant (1) } \\
\text { s'informatiser }\end{array}$} & \multicolumn{2}{|c|}{ Informatisées } & \multicolumn{2}{|l|}{ Total } \\
\hline & & & Nombre & $\%$ & Nombre & $\%$ & Nombre & $\%$ & Nombre & $\%$ \\
\hline 0 & à & 9 employés & 95 & 28,4 & 69 & 20,1 & 171 & 51,0 & 335 & 100,0 \\
\hline 10 & à & 49 & 97 & 13,5 & 102 & 14,2 & 520 & 71,3 & 719 & 100,0 \\
\hline 50 & à & 99 & 7 & 2,9 & 23 & 9,4 & 214 & 87,7 & 244 & 100,0 \\
\hline 100 & à & 299 & 1 & 0,6 & 7 & 4,5 & 149 & 94,9 & 157 & 100,0 \\
\hline 300 & à & 499 & - & - & 1 & 0,8 & 125 & 99,2 & 126 & 100,0 \\
\hline 500 & à & 999 & - & - & - & - & 47 & 100,0 & 47 & 100,0 \\
\hline \multirow[t]{2}{*}{$1000 e$} & et & plus & - & - & - & - & 78 & 100,0 & 78 & 100,0 \\
\hline & & TOTAL & 200 & & 202 & & 1304 & & 1706 & (2) \\
\hline
\end{tabular}

(1) Dans les douze prochains mois.

(2) Parmi les 1708 entreprises, deux entreprises n'ont pu être identifiées à une taille.

Ces résultats permettent de valider partiellement notre première proposition selon laquelle le chiffre d'affaires pour une même taille d'entreprise serait associé significativement à la décision d'informatisation. En effet, les valeurs médianes du chiffre d'affaires sont systématiquement plusélevées pour lesentreprises informatisées ou prévoyant s'informatiser. Les différences intergroupes ne sont, par contre, pas toujours significatives. 


\section{Tab. 3. Résultats du test de P1}

\begin{tabular}{|c|c|c|c|c|c|c|}
\hline \multirow{2}{*}{\multicolumn{2}{|c|}{ Taille de l'entreprise }} & \multicolumn{3}{|c|}{$\begin{array}{l}\text { Chiffre d'affaires médian pour les entreprises } \\
\text { (en milliers de \$) }\end{array}$} & \multicolumn{2}{|c|}{$\begin{array}{l}\text { Niveau critique } \\
\text { observé (1) }\end{array}$} \\
\hline & & $\begin{array}{c}\text { Ne prévoyant pas } \\
\text { s'informatiser } \\
\text { Md1 }\end{array}$ & $\begin{array}{l}\text { Prévoyant } \\
\text { s'informatiser } \\
\text { Md2 }\end{array}$ & $\begin{array}{l}\text { Informatisées } \\
\text { Md3 }\end{array}$ & P1 & P2 \\
\hline 0 & 9 emplovés & 300 & 100 & 500 & 0,636 & $0,035^{*}$ \\
\hline & & 1000 & 2000 & 2500 & 0,341 & $0,002^{*}$ \\
\hline 50 & 99 & 3000 & 6000 & 75000 & 1,000 & 0,481 \\
\hline \multicolumn{2}{|c|}{$\begin{array}{l}\text { Toutes les petites } \\
\text { entreprises }\end{array}$} & 750 & 1500 & 5000 & $0,000^{*}$ & $0,000^{*}$ \\
\hline
\end{tabular}

(1) Test des médianes p1 = niveau critique observée pour $M d 1<M d 2$ p2 = niveau critique observé pour Md2 $<M d 3$

- Significatif pour $p<0,05$

Les propositions 2 et 3 portent sur les dépenses actuelles et prévues pour l'acquisition d'équipements et de logiciels. Au tableau 4, les résultats indiquent que les petites entreprises consacrent de façon significative un pourcentage proportionnellement plus important de leur chiffre d'affaires à l'acquisition d'équipements informatiques (P2). Il en est de même des coûts d'acquisition prévus (P3).

\section{Tab. 4. Résultats des tests de P2 et P3}

\begin{tabular}{|l|c|c|c|c|c|}
\cline { 2 - 6 } \multicolumn{1}{c|}{} & \multicolumn{3}{c|}{ Taille de l'entreprise } & \multicolumn{2}{c|}{$\begin{array}{c}\text { Niveau critique } \\
\text { observé (1) }\end{array}$} \\
\hline Ratio médian & $\begin{array}{c}\text { Petite } \\
\text { entreprise } \\
\text { Md1 }\end{array}$ & $\begin{array}{c}\text { Entreprise } \\
\text { moyenne } \\
\text { Md2 }\end{array}$ & $\begin{array}{c}\text { Grande } \\
\text { entreprise } \\
\text { Md3 }\end{array}$ & $p 1$ & $p 2$ \\
\hline $\begin{array}{l}\text { Dépenses actuelles } \\
\text { (matériel et } \\
\text { logiciel) / chiffre } \\
\text { d'affaires annuel } \\
\text { (P2) }\end{array}$ & 1,33 & 0,83 & 0,83 & $0,000^{*}$ & $0,004^{*}$ \\
\hline $\begin{array}{l}\text { Investissements } \\
\text { prévus) / chiffre } \\
\text { d'affaires annuel } \\
\text { (P3) }\end{array}$ & 0,33 & 0,25 & 0,18 & $0,000^{*}$ & $0,002^{*}$ \\
\hline
\end{tabular}

(1) Test des médianes p1 = niveau critique observé pou Md1 > Md2 p2 = niveau critique observé pour Md1 $>$ Md3

- Significatif pour $p<0,05$ 
En regard des frais annuels d'exploitation, nos résultats indiquent que peu de petites entreprises accordent de façon formelle un budget annuel pour les frais d'exploitation de leur système informatique. Au total, $63 \%$ d'entre elles ont un budget pour l'entretien, $43 \%$ pour la formation, et seulement $19 \%$ pour les mesures de sécurité. Par contre, pour celles qui ont un budget annuel accordé à un type de frais d'exploitation, le fardeau financier est relativement plus élevé que celui retrouvé dans les moyennes et grandes entreprises. (tableau 5).

Tab. 5. Résultats du test de P4

\begin{tabular}{|c|c|c|c|c|c|}
\hline \multirow[b]{2}{*}{ Ratio médian } & \multicolumn{3}{|c|}{ Taille de l'entreprise } & \multicolumn{2}{|c|}{$\begin{array}{c}\text { Niveau critique } \\
\text { observé (1) }\end{array}$} \\
\hline & $\begin{array}{l}\text { Petite } \\
\text { entreprise } \\
\text { Md1 }\end{array}$ & $\begin{array}{l}\text { Entreprise } \\
\text { moyenne } \\
\text { Md2 }\end{array}$ & $\begin{array}{l}\text { Grande } \\
\text { entreprise } \\
\text { Md3 }\end{array}$ & P1 & P2 \\
\hline $\begin{array}{l}\text { Budget annuel accordé } \\
\text { à la formation/chiffre } \\
\text { d'affaires annuel }\end{array}$ & 0,067 & 0,027 & 0,015 & $0,000^{*}$ & $0,000^{*}$ \\
\hline $\begin{array}{l}\text { Budget annuel accordé } \\
\text { à l'entretien/ } \\
\text { chiffre d'affaires annuel }\end{array}$ & 0,107 & 0,056 & 0,067 & $0,000^{*}$ & 0,230 \\
\hline $\begin{array}{l}\text { Budget annuel accordé } \\
\text { à la sécuritél } \\
\text { chiffre d'affaires annuel }\end{array}$ & 0,022 & 0,016 & 0,007 & 0,200 & $0,002^{*}$ \\
\hline
\end{tabular}

(1) Test des médianes

p1 = niveau critique observé pou Md1 $>$ Md2

p2 = niveau critique observé pour Md1 > Md3

- Significatif pour $p<0,05$

\section{Discussion et conclusion}

Il a été démontré que la décision d'informatisation semble être étroitement liée au chiffre d'affaires des petites entreprises, qu'elles soient artisanales ou autres. Or, le chiffre d'affaires est généralement associé au volume d'activité d'une entreprise et, à ce titre, peut constituer un des indicateurs des besoins d'informatisation.

Au-delà de cette première constatation, on a aussi remarqué que le fardeau financier associé à l'informatisation est proportionnellement plus élevé dans la petite entreprise que dans la moyenne et la grande. En effet, un pourcentage plus élevé du chiffre d'affaires est consacré aux coûtsd'acquisition et aux frais annuels d'exploitation de ces technologies. 
Considérant l'importance des dépenses relatives à l'informatisation pour la petite entreprise, il apparât essentiel qu'elles soient bien planifiées. Dans ce contexte, on comprend bien l'importance accordée à la planification et au lien établi entre cette dernière et la survie de la firme (Robinson, 1979; Stoner, 1983; Kelley et Young, 1983). Cette préoccupation est d'autant plus justifiée si on tient compte de la difficulté pour les petites entreprises de planifier adéquatement les investissements requis pour l'informatisation (Malone, 1985; Lees et Lees, 1987; Lefebvre et Lefebvre, 1988). Or des recherches récentes (Lefebvre et Lefebvre, 1988b) ont démontré que peu de petites entreprises planifiaient l'acquisition et l'introduction de l'informatique entraînant des conséquences souvent désastreuses sur le plan financier.

En conclusion, compte tenu que le fardeau financier de l'informatisation est plus important dans la petite entreprise et que les risques sont plus élevés, la planification financière requise lors de l'acquisition et de l'introduction de l'informatique dansl'entreprise serait fortement souhaitable, sinon essentielle. Cette préoccupation devient primordiale si l'on considère le nombre important de petites entreprises qui s'informatiseront ou poursuivront leur processus d'informatisation dans les prochaines années.

\section{BIBLIOGRAPHIE}

Conseil économique du Canada, (1987), «Le recentrage technologique : innovation, emplois, adaptations», Ottawa, Approvisionnements et Services

Conseil économique du Canada, (1986), «La technologie en milieu de travail - Enquête sur l'automatisation au Canada», Ottawa, Approvisionnements et Services.

DeLone, W.H., (1981), «Firm size and the characteristics of computer use», MIS Quarterly, vol. $5, \mathrm{n}^{\circ} 4$, décembre.

DeLone, W.H., (1988), «Determinants of success for computer usage in small business», MIS Quarterly, vol. 12, no 1, mars.

Griffith, J. et Dorsman, M., (1986), «SME's new technology, and training», International Small Business Journal, vol. 5, $\mathrm{n}^{\circ}$ 3, 1986.

Julien, P.-A., Carrière, J.-B. et Hébert, L., (1988), «La diffusion des nouvelles technologies dans trois secteurs industriels», Conseil de la science et de la technologie, document no 88 03, Québec, avril.

Lees, J.D., (1987), «Successful development of small business information systems», Journal of Systems Management, septembre.

Lees, J.D. et Lees, D.D., (1987), «Realities of small business information system implementation», Journal of Systems Management, janvier.

Kelley, C.A. et Young, J.E., (1983), «Is our small business ready or planning ?», Journal of Small Business Management, janvier.

Lefebvre, L.A., Lefebvre, E., Ducharme, J. et Colin, D., (1986), «L'impact de la technologie informatique sur la main-d'oeuvre dans les organisations», L'Actualité économique, vol. $62, n^{\circ} 4$. 
Lefebvre, L.A. et Lefebvre, E., (1988a), «The importance of planning computer acquisitions», document de travail $n^{\circ}$ 05-88, Centre de recherche en Gestion, UQAM.

Lefebvre, L.A. et Lefebvre, E., (1988b), «Computerization of small firms : a study of the perceptions and expectations of managers», Journal of Small Business \& Entrepreneurship, vol. $5, n^{\circ} 5$, été.

Malone, S.C., (1985), «Computerizing small business information systems», Journal of Small Business Management, avril.

Martin, J. et McClure, C., (1983), «Buying software off the rack», Harvard Business Review, novembre-décembre.

Meredith, J., (1987), «The strategic advantages of new manufacturing technologies for small firms», Strategic Management Journal, vol. 8.

MIC (Ministère de l'Industrie et du Commerce), (1987), «Les PME au Québec. Etat de la situation», Gouvernement du Québec, Québec.

OTA (Office of Technology Assessment), (1985), «Automation of America's offices 19852000», Congress of the United States.

Raymond, L., (1985), «Organization characteristics and MIS success in the context of small business», MIS Quarterly, vol. 9, $\mathrm{n}^{\circ} 1$.

Robinson, R., (1979), «Forecasting and small business : a study of the strategic planning process», Journal of Small Business Management, juillet.

Stoner, C.R., (1983), «Planning in small manufacturing firms : a survey», Journal of Small Business Management, janvier.

Welsh, J.A. \& White, J.F., (1981), «A small business is not a little big business», Harvard Business Review. 